\title{
Microstructural evolution, atomic migration, and FePt nanoparticle formation in ion-irradiated $\mathrm{Pt}(\mathrm{Fe}) / \mathrm{C}(\mathrm{Fe})$ multilayers
}

\author{
S. Bera, S. Roy, and K. Bhattacharjee \\ Institute of Physics, Sachivalaya Marg, Bhubaneswar,751005, India \\ G. Kuria) \\ Hamburg Synchrotron Radiation Laboratory (HASYLAB) at DESY, Notkestrasse 85, D-22603 Hamburg, \\ Germany \\ B. N. Dev ${ }^{\text {b) }}$ \\ Institute of Physics, Sachivalaya Marg, Bhubaneswar, 751005, India \\ and \\ Department of Materials Science, Indian Association for the Cultivation of Science, $2 A$ and $2 B$ Raja S. C. \\ Mullick Road, Jadavpur, Kolkata 700032, India
}

(Received 10 April 2007; accepted 23 May 2007; published online 11 July 2007)

\begin{abstract}
Ion-irradiation-induced microstructural modifications, atomic migration, and nanoparticle formation in nanoscale periodic Pt/C multilayer systems containing about 15 at. \% Fe have been analyzed by $\mathrm{X}$-ray reflectivity, $\mathrm{X}$-ray standing wave (XSW), transmission electron microscopy, and grazing incidence X-ray diffraction (GIXRD) studies. The multilayer stack with $15 \mathrm{Pt}(\mathrm{Fe}) / \mathrm{C}(\mathrm{Fe})$ layer pairs (period $4.2 \mathrm{~nm}$ ) was irradiated with a $2 \mathrm{MeV} \mathrm{Au}{ }^{2+}$ ion beam, which was rastered on the sample to obtain uniformly irradiated strips with fluences $(\phi)$ from $1 \times 10^{14}$ to $1 \times 10^{15}$ ions $/ \mathrm{cm}^{2}$. Up to a certain threshold ion fluence $\left(\phi_{t}\right)$ we have observed mixing between $\mathrm{Pt}$ and $\mathrm{C}$ atoms. At higher fluences $\left(\phi>\phi_{t}\right)$ demixing effect is pronounced. This phenomenon has been explained in terms of two competitive processes including ballistic and chemically guided atomic movements. As revealed by XSW measurements, ion irradiation causes preferential migration of Fe toward Pt layers from C layers. Consequently Fe concentration in the Pt layers increases with a corresponding decrease in the $\mathrm{C}$ layers. The decrease in $\mathrm{Fe}$ concentration in the $\mathrm{C}$ layers has an exponential dependence on the ion fluence. From the gradual change in shape of the Fe fluorescence yield curve, over the first order Bragg peak region, due to ion irradiation we can infer that Fe atoms migrate from $\mathrm{C}$ to Pt layers in the outward direction. This outward migration of Fe has been explained in terms of surface segregation of Fe in $\mathrm{Pt}$ under the irradiation condition. Cross-sectional transmission electron microscopy has revealed the gradual change in the microstructure, considerable atomic redistribution, and cluster formation for irradiation at the highest ion fluence $\left(1 \times 10^{15}\right.$ ions $\left./ \mathrm{cm}^{2}\right)$, where FePt nanoparticle formation is observed in GIXRD experiments. The results and analysis presented in this work provide an understanding of the evolution of ion-beam-induced ferromagnetism and the increasing coercive field with ion fluence in the $\mathrm{Pt}(\mathrm{Fe}) / \mathrm{C}(\mathrm{Fe})$ multilayer system [B. N. Dev et al., Microelectronic Eng. 83, 1721 (2006)]. (C) 2007 American Institute of Physics. [DOI: 10.1063/1.2752150]
\end{abstract}

\section{INTRODUCTION}

Layered synthetic microstructures (LSMs) are fabricated by depositing alternating layers of usually two different materials on a substrate. The LSMs have interesting structural, ${ }^{1}$ magnetic, ${ }^{2}$ and electronic ${ }^{3}$ properties with a wide range of applications. LSMs containing alternating layers of high atomic number elements (e.g., Pt, W, etc.) and low atomic number elements (e.g., C, Si, etc.) are being used as X-ray reflectors. X-ray multilayer optics is now used in X-ray astronomy, microscopy, and spectroscopy, and as filters and monochromators for intense sources such as synchrotron radiation and x-ray laser cavities. Magnetic multilayers are also used for magnetic recording heads and sensors. It is

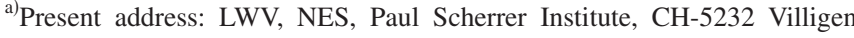
PSI, Switzerland.

${ }^{b)}$ Electronic mail: bhupen@iopb.res.in; msbnd@iacs.res.in
}

important to correlate the measured properties with structure so that preparation techniques can be optimized to get high performance materials. The composition of individual layers, their relative thickness, surface roughness, and interface roughness play important roles in multilayer performance. Properties of such multilayers can also be modified by introducing impurities or by exposing to energetic ion beams. Magnetic multilayers, e.g., $\mathrm{Co} / \mathrm{Cu}$ multilayers, have shown drastic changes in magnetic coupling and magnetoresistance in the presence of small magnetic impurity (e.g., Ni) concentrations in the nonmagnetic $(\mathrm{Cu})$ layers. ${ }^{4}$ Such small concentrations of magnetic impurity can be easily introduced from the magnetic to nonmagnetic layers by ion irradiation. ${ }^{5}$ Various research groups have revealed change of the giant magnetoresistance in $\mathrm{Co} / \mathrm{Cu}$ multilayers by annealing and by ion irradiation. ${ }^{6-10}$ Ion beam irradiation has been shown to rotate the easy magnetization direction from out-of-plane to in- 
plane in $\mathrm{Co} / \mathrm{Pt}$ multilayers. These changes are due to the structural reordering at the multilayer interfaces and are accompanied by an increase in grain size. ${ }^{11-20}$ From these examples it is clear that atomic distribution and ion beam induced atomic redistribution in multilayers are important aspects to be investigated for an understanding of the multilayer properties. When the individual layer thickness in a multilayer is of the order of $1 \mathrm{~nm}$, standard techniques like conventional Rutherford backscattering spectrometry, secondary ion mass spectrometry, etc. are unsuitable for the determination of atomic depth distribution due to the limitation on resolution of these techniques. However, a combined $\mathrm{x}$-ray standing wave (XSW) and reflectivity (XRR) analysis has been shown to be capable of determining this distribution in such multilayers. ${ }^{5,21}$

In the present article we report a detailed analysis of combined XSW and XRR measurements for the study of ion-irradiation-induced change in composition in individual layer, multilayer period, surface roughness and interface roughness, and $\mathrm{Pt}$ and $\mathrm{Fe}$ atomic redistributions in a $\mathrm{Pt} / \mathrm{C}$ periodic multilayer system containing a small amount of Fe. The Pt/C multilayer system has been chosen as a model system as the large electron density contrast between Pt and C gives rise to strong Bragg diffraction peaks from a periodic $\mathrm{Pt} / \mathrm{C}$ multilayer. This in turn generates a strong standing wave field in the multilayer.

Depending on the ion, its energy, and fluence, ionbombardment can enhance atomic diffusion and is thus capable of modifying the microstructure of the $\mathrm{Pt} / \mathrm{C}$ multilayers. Such changes are strongly dependent on the details of the chemical interactions between the constituents, their miscibility, and heat of mixing. Additionally, ion irradiated Pt/C multilayers with $\mathrm{Fe}$ [hereafter denoted by $\mathrm{Pt}(\mathrm{Fe}) / \mathrm{C}(\mathrm{Fe})]$ may themselves show interesting magnetic behavior, as there are possibilities of formation of Fe-Pt magnetic alloys, possibly as small clusters. Carbon can provide magnetic isolation between neighboring clusters, which causes the reduction of interparticle exchange coupling. In fact we have observed magnetic domain structure in the sample irradiated at the highest fluence. ${ }^{22}$

\section{EXPERIMENT}

$\mathrm{Pt} / \mathrm{C}$ multilayers were fabricated on float glass substrates, by ion beam sputtering, at a low argon pressure of 0.1 mbar. A small amount of $\mathrm{Fe}$ (about 15 at. $\%)^{23}$ was introduced in the multilayers during growth. The sample is denoted hereafter as $\mathrm{Pt}(\mathrm{Fe}) / \mathrm{C}(\mathrm{Fe})$. The $\mathrm{Pt}(\mathrm{Fe}) / \mathrm{C}(\mathrm{Fe})$ multilayer sample used in this study, was prepared at Nagoya University. The sample specifications are: $N=15$ (the number of layer pairs in the multilayer stack), $d=4.2 \mathrm{~nm}$ (multilayer period, i.e., the thickness of a Pt/C layer pair), and $\Gamma=0.38$ (ratio of Pt layer thickness to $d$ ). The total thickness of the multilayer stack is about $63 \mathrm{~nm}$. Different parts of a large sample $(30 \times 70) \mathrm{mm}^{2}$ were irradiated with $2 \mathrm{MeV} \mathrm{Au}^{2+}$ ions by rastering the ion beam on $(30 \times 5) \mathrm{mm}^{2}$ strips at various fluences (ions $\left./ \mathrm{cm}^{2}\right)\left[\mathrm{V}\right.$ (virgin), B $\left(1 \times 10^{14}\right), \mathrm{C}(3$ $\left.\left.\times 10^{14}\right), \mathrm{D}\left(5 \times 10^{14}\right), \mathrm{E}\left(7 \times 10^{14}\right), \mathrm{F}\left(1 \times 10^{15}\right)\right]$ at Institute of Physics, Bhubaneswar. The range of $2 \mathrm{MeV}$ Au ions
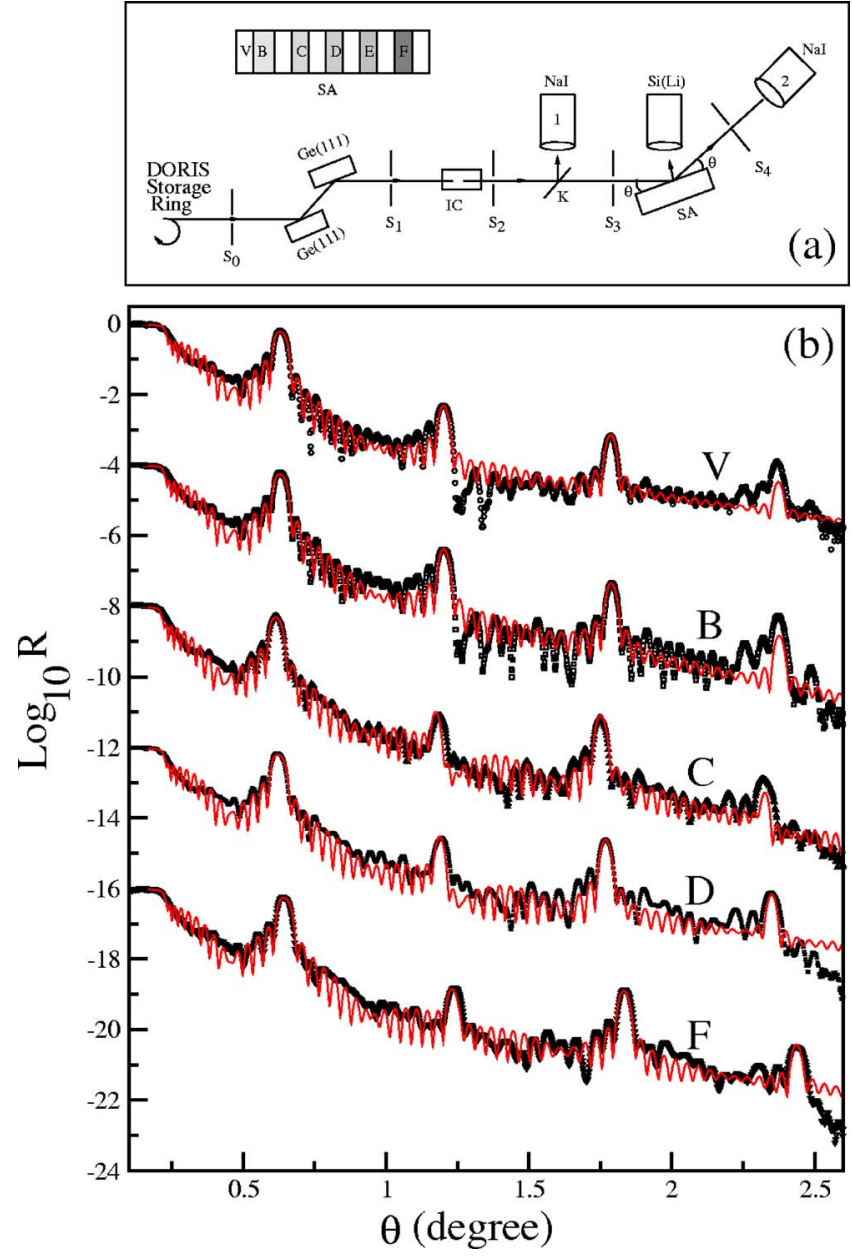

FIG. 1. (a) The experimental setup for the x-ray reflectivity and standing wave measurements and the samples are schematically shown. (b) Experimental $\mathrm{x}$-ray reflectivity data from the virgin, $\mathrm{V}$ (circle), and the ionirradiated, B-F (different symbols) multilayer samples and the fitted theoretical reflectivity curves (solid curves) for a $\mathrm{Pt}(\mathrm{Fe}) / \mathrm{C}(\mathrm{Fe})$ multilayer with 15 bilayers on a glass substrate. Data for the irradiated samples are vertically shifted down for clarity. $\mathrm{B} \rightarrow \mathrm{F}$ represent increasing ion fluence (in ions $\left./ \mathrm{cm}^{2}\right)$ : B $\left(1 \times 10^{14}\right), \mathrm{C}\left(3 \times 10^{14}\right), \mathrm{D}\left(5 \times 10^{14}\right)$, and $\mathrm{F}\left(1 \times 10^{15}\right)$.

in such Pt/C multilayer samples is about $270 \mathrm{~nm}$. Thus the implanted $\mathrm{Au}$ ions are buried deep into the glass substrate having passed through the multilayer stack, which is about $63 \mathrm{~nm}$ thick. The virgin and the irradiated strips were analyzed with $\mathrm{x}$-ray standing wave and $\mathrm{x}$-ray reflectivity experiments. X-ray standing wave and reflectivity experiments were carried out at Hamburg Synchrotron Radiation Laboratory (HASYLAB at DESY), Hamburg, at the ROEMO-I beamline using $14.0 \mathrm{keV}$ monochromatized $\mathrm{x}$ rays. A monocromatic beam $(\lambda=0.89 \AA)$ was obtained using a double crystal monochromator with $\mathrm{Ge}(111)$ crystals. Pt $L$ and $\mathrm{Fe} K$ fluorescent photons were detected by a $\mathrm{Si}(\mathrm{Li})$ detector that was placed at an angle of $15^{\circ}$ with respect to the sample surface and the reflectivity was measured by a $\mathrm{NaI}$ detector. The sample configuration (top view) and the schematic diagram of the x-ray experimental setup are shown in Fig. 1(a). Cross-sectional transmission electron microscopy (XTEM) measurements on the irradiated samples (D, E, and F) were carried out with $200 \mathrm{keV}$ electrons using a JEOL 2010(UHR) electron microscope at Institute of Physics. 
TABLE I. Results of XRR and XSW measurements on the virgin and the irradiated $\mathrm{Pt}(\mathrm{Fe}) / \mathrm{C}(\mathrm{Fe})$ multilayers. Typical error bars for layer thickness and roughness are less than $1 \AA$ and those on coherent fractions are \pm 0.02 .

\begin{tabular}{|c|c|c|c|c|c|c|c|c|c|c|}
\hline $\begin{array}{c}\mathrm{Pt}(\mathrm{Fe}) / \mathrm{C}(\mathrm{Fe}) \\
\text { sample }\end{array}$ & $\begin{array}{c}\text { Pt layer } \\
\text { density } \\
\left(\mathrm{e} / \AA^{3}\right)\end{array}$ & $\begin{array}{l}\text { C layer } \\
\text { density } \\
\left(\mathrm{e} / \AA^{3}\right)\end{array}$ & $\begin{array}{c}\text { Pt layer } \\
\text { thickness } \\
d_{1}(\AA)\end{array}$ & $\begin{array}{c}\text { C layer } \\
\text { thickness } \\
d_{2}(\AA)\end{array}$ & $\begin{array}{c}\text { Multilayer } \\
\text { period } \\
d(\AA)\end{array}$ & $\begin{array}{c}\text { Surface } \\
\text { roughness } \\
\sigma_{0}(\AA)\end{array}$ & $\begin{array}{c}\text { Pt-on-C } \\
\text { interface } \\
\text { roughness } \\
\sigma_{1}(\AA)\end{array}$ & $\begin{array}{c}\text { C-on-Pt } \\
\text { interface } \\
\text { roughness } \\
\sigma_{2}(\AA)\end{array}$ & $\begin{array}{l}\text { Pt coherent } \\
\text { fraction }\end{array}$ & $\begin{array}{c}\mathrm{Fe} \text { coherent } \\
\text { fraction }\end{array}$ \\
\hline $\mathrm{V}$ & 3.8 & 0.8 & 20.4 & 22.5 & 42.9 & 2.5 & 4.1 & 3.8 & 0.93 & 0.55 \\
\hline B & 3.6 & 0.8 & 20.4 & 22.5 & 42.9 & 2.9 & 4.3 & 3.9 & 0.92 & 0.59 \\
\hline $\mathrm{C}$ & 3.1 & 0.8 & 20.9 & 22.8 & 43.7 & 2.9 & 4.6 & 3.9 & 0.83 & 0.75 \\
\hline $\mathrm{D}$ & 3.6 & 0.8 & 20.8 & 22.5 & 43.3 & 1.8 & 4.0 & 3.1 & 0.87 & 0.82 \\
\hline F & 3.5 & 0.8 & 21.4 & 20.4 & 41.8 & 1.9 & 4.3 & 3.2 & 0.87 & 0.88 \\
\hline
\end{tabular}

\section{RESULTS AND DISCUSSIONS}

\section{A. Microstructures}

The experimental reflectivity data and the fitted theoretical reflectivity curves for both the virgin and the irradiated multilayer samples are shown in Fig. 1(b). Strong peaks in each set of data are first, second, third, and fourth order Bragg peaks from the periodic multilayers satisfying the Bragg's law: $2\left(d_{1} \sin \theta_{1}+d_{2} \sin \theta_{2}\right)=n \lambda$, where the period of the multilayer is $d=d_{1}+d_{2}, d_{1}$ and $d_{2}$ being the thickness of a Pt and C layer, respectively, and $n$ is the order of reflection. The small oscillation interference fringes are due to the total thickness of the multilayer stack. The beating pattern (clearly visible for samples $\mathrm{V}$ and $\mathrm{B}$ ) manifested in reduced amplitude of oscillation between Bragg peaks are due to a thicker Pt layer $\left(>d_{1}\right)$ at the bottom, as observed later in the XTEM micrograph in Fig. 4, as well as in the XRR simulation. The experimental data in Fig. 1(b) have been fitted by allowing the variation in the electron density, layer thickness, surface roughness, and interface roughness of the layers. Results are listed in Table I. The thickness of the first Pt layer on the glass substrate is $81 \AA$ as obtained from the best fit. The experimental first order Bragg peaks $[R(\theta)]$ from the virgin and the irradiated samples are shown in Fig. 2. From Fig. 2 and Table I we notice that, for the irradiated sample B, there is no change in $d$ spacing (multilayer period) compared to the virgin sample $\mathrm{V}(d=42.9 \AA)$ but there is a slight reduction in the first order Bragg peak reflectivity from 0.65 to

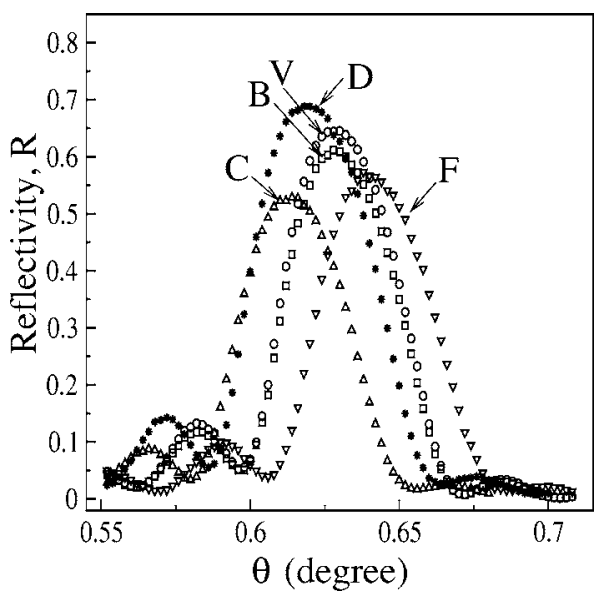

FIG. 2. The first order Bragg peaks are shown in the linear scale from the virgin (open circle) and the irradiated samples: B (square), C (triangle up), D (star), and $\mathrm{F}$ (triangle down).
0.62. Sample $\mathrm{C}$ shows expansion of the multilayer period $\left(\begin{array}{ll}d=43.7 \AA & \AA\end{array}\right)$ and further reduction in the first order peak reflectivity $(R=0.52)$ compared to sample B $(d=42.9 \AA$ and $R=0.62$ ). This trend of period dilation and reflectivity reduction with increasing ion fluence was also observed earlier up to a fluence of $1 \times 10^{14}$ ions $/ \mathrm{cm}^{2}$ for $\mathrm{Pt} / \mathrm{C}$ multilayers irradiated with $2 \mathrm{MeV}$ Au ions. ${ }^{5} 1 \times 10^{14}$ ions $/ \mathrm{cm}^{2}$ is the maximum ion fluence used in Ref. 5. At a higher ion fluence of $5 \times 10^{14}$ ions $/ \mathrm{cm}^{2}$ we observe contraction (with respect to sample $\mathrm{C}$ ) in the multilayer period for sample $\mathrm{D}$, for which $d=43.3 \AA$, and there is an increase in peak reflectivity, $R$ $=0.69$. (We have not carried out XRR and XSW experiments on sample $\mathrm{E}$ due to the lack of beam time during the experiment at the Synchrotron source.) There is further contraction in $d$ spacing $(d=41.8 \AA)$ and the Bragg peak reflectivity reduces to 0.59 in sample $\mathrm{F}$, which was irradiated at a fluence of $1 \times 10^{15}$ ions $/ \mathrm{cm}^{2}$. So ion irradiation up to a fluence of $3 \times 10^{14}$ ions $/ \mathrm{cm}^{2}$ causes multilayer expansion. Increasing ion fluence beyond this value has been found to cause a contraction of the multilayer period.

A plot of first, second, third, and fourth order Bragg peak intensities as a function of irradiation fluence is shown in Fig. 3. Irradiation induces significant changes in the Bragg peak reflectivity. Bragg peak intensities for all orders initially decrease, then increase, and again decrease with increasing ion fluence (Fig. 3). In order to understand these decrease or

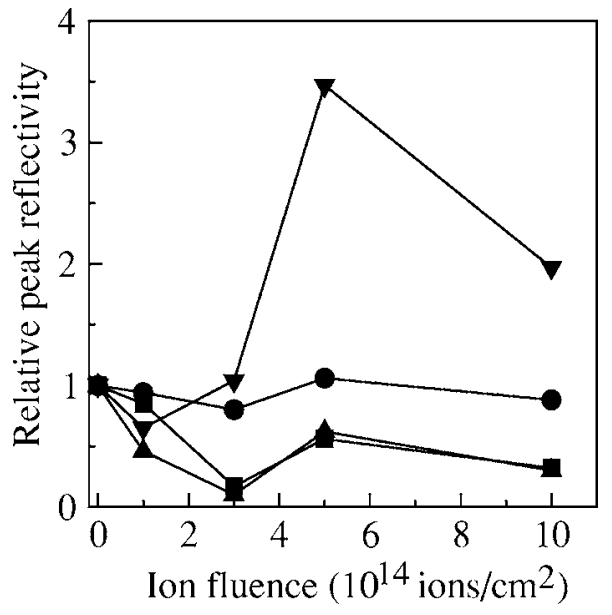

FIG. 3. Relative reflectivity of Bragg peaks for the irradiated multilayers normalized to the value for the as-deposited multilayer vs irradiation fluence: First (filled circle), second (filled square), third (filled triangle down), and fourth (filled triangle up) Bragg peak. 
increase in Bragg peak reflectivity upon ion irradiation, the following aspects are to be considered: (a) the change in multilayer period, (b) the change in optical contrast between the constituent materials, (c) the sharpening or broadening of the interfaces, and (d) the change in lateral uniformity.

These results can be understood in conjunction with the results obtained from the XRR analysis presented in Table I. At relatively low fluence irradiation, as in Ref. 5, ballistic mixing across the $\mathrm{Pt} / \mathrm{C}$ interfaces increase interface roughness. Pt atoms are pushed into $\mathrm{C}$ layers and vice versa. This decreases the electron density of the Pt layers seen in Table 1. The electron density of the C layers would increase. However, the reflectivity is not very sensitive to small changes in the electron density of the $\mathrm{C}$ layers. ${ }^{21}$ That is why we kept this parameter fixed in fitting the data. Ballistic mixing also causes an expansion of the multilayer period. ${ }^{5}$ The reduction in the electron density contrast between Pt and C layers lead to reduced Bragg peak reflectivity. At a higher fluence, say from samples $C$ to $D$, as we see from Table I, surface and interface roughnesses decrease and electron density of the $\mathrm{Pt}$ layers increase. This is due to demixing across the $\mathrm{Pt} / \mathrm{C}$ interfaces. Although at low fluence ballistic mixing occurs, at higher fluences, whether mixing will be aided or demixing will occur depends on whether the components of the multilayer (here $\mathrm{Pt}$ and $\mathrm{C}$ ) are miscible or immiscible, i.e., on the heat of mixing. The heat of mixing between $\mathrm{Pt}$ and $\mathrm{C}$ is strongly positive $\left(\Delta H_{m}^{1: 1}=52.7 \mathrm{~kJ} / \mathrm{mol}\right) ;{ }^{24}$ so there is no tendency for alloying. This chemical drive leads to demixing at higher ion fluences. The electron density contrast increases due to demixing and this leads to an increase in the Bragg peak reflectivity. This trend reversal from samples $C$ to $D$ is observed in all parameters (Table I) except the one in the last column, which does not directly involve the components of the multilayer. From Table I, we notice that for sample F several parameters look anomalous. There are minor changes in the electron density and surface and interface roughnesses compared to sample D. However, the multilayer period and the layer thicknesses have large changes. The multilayer period has contracted even in comparison to the virgin sample $\mathrm{V}$. These results cannot be explained from the line of arguments presented earlier. Inputs from other experiments are necessary to explain these results. XTEM results provide such input.

In order to study the microstructural changes from samples D to F we have carried out XTEM measurements on samples D, E, and F. Cross-sectional TEM images are shown in Fig. 4. From Fig. 4 it is clear that in sample D, the Pt and the $\mathrm{C}$ layers preserve their identity as continuous layers and the interfaces are smooth. XTEM image of sample E shows that layers begin to break and become discontinuous in the multilayer irradiated at an ion fluence of $7 \times 10^{14}$ ions $/ \mathrm{cm}^{2}$. XTEM image of sample $\mathrm{F}$ shows major atomic redistribution, phase separation, and cluster formation. This major change in the microstructure is responsible for the large change and anomalous behavior in the parameters extracted from the XRR analysis (Table I). Transmission electron diffraction patterns from sample $\mathrm{F}$ shows diffraction spots, corresponding to new length scales, in addition to those expected from a periodic multilayer. ${ }^{24}$ In fact, $\mathrm{FePt}$

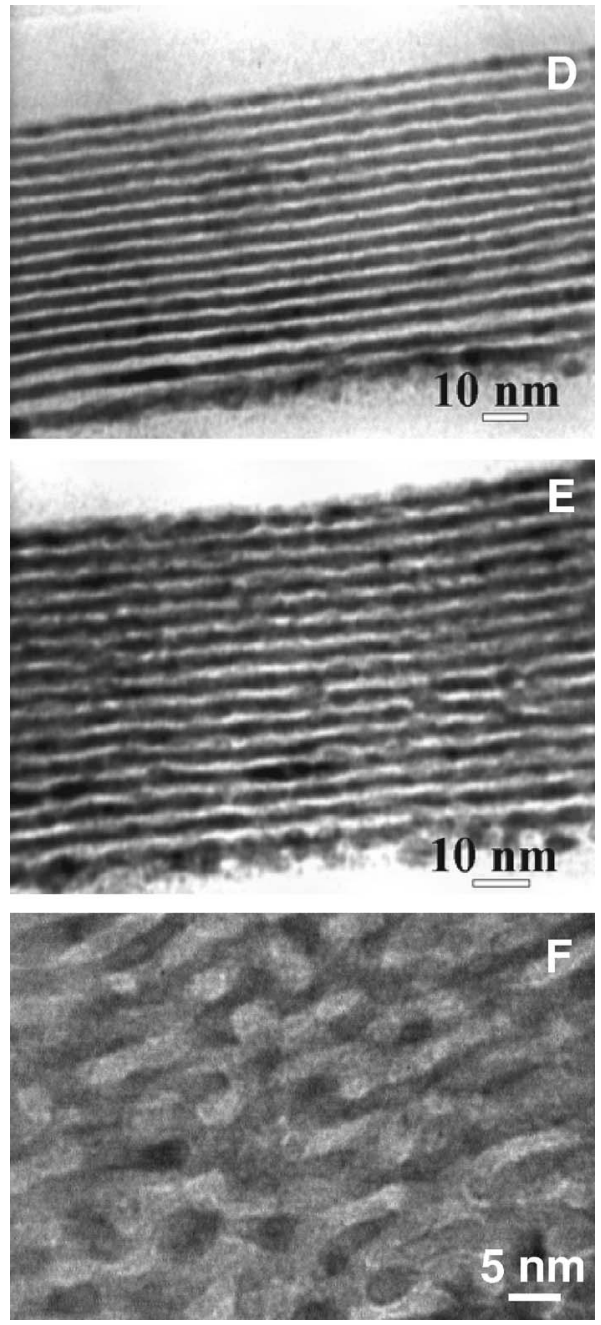

FIG. 4. Cross-sectional TEM images of the irradiated samples D (fluence: $5 \times 10^{14}$ ions $/ \mathrm{cm}^{2}$ ), E (fluence: $7 \times 10^{14}$ ions $/ \mathrm{cm}^{2}$ ), and $\mathrm{F}$ (fluence: 1 $\times 10^{15}$ ions $/ \mathrm{cm}^{2}$ ).

nanoparticles surrounded by $\mathrm{C}$ have been formed in sample F. Glancing incidence x-ray diffraction (GIXRD) results confirm the formation of FePt. The mechanism of FePt formation will be discussed in Sec. III B.

Ion irradiation displaces atoms via ion-atom collisions and in turn introduces atoms from one layer into another- $\mathrm{Pt}$ into $\mathrm{C}$ and $\mathrm{C}$ into $\mathrm{Pt}^{5,21}$ Determination of accurate atomic concentration and change of atomic concentration due to ion irradiation in the layers is difficult from the reflectivity results. Especially, in the $\mathrm{C}$ layers, large changes (even up to $15 \%$ change) in the electron density of the $\mathrm{C}$ layers are practically indistinguishable from the reflectivity. ${ }^{21}$ That is why we did not vary C-layer density to fit the reflectivity curves. Thus from the analysis of XRR data, the effect of Pt introduction into $\mathrm{C}$ layers cannot be reliably extracted. In our XRR analysis we fixed the electron density of the $\mathrm{C}$ layers to be $0.8 \mathrm{e} / \AA^{3}$, which is slightly higher than that of pure C. In the present system it is further complicated by the initial presence of $\mathrm{Fe}$ in the $\mathrm{Pt}$ and $\mathrm{C}$ layers and ion beam induced redistribution of $\mathrm{Fe}$. Layer compositions are more accurately determined by $\mathrm{x}$-ray standing wave measurements. Each Bragg diffraction peak is associated with a standing wave pattern of $\mathrm{x}$ rays within the multilayer. We have used the first 
order Bragg peak, for which the standing wave field is strong, for the detection of Pt and Fe distribution within the multilayer. The details of this kind of analysis are available in Refs. 5 and 21. The standing wave field has the same period as the period of the multilayer. At the rising edge of Bragg reflection the antinodes of the standing wave field are within the $\mathrm{C}$ layers. As the angle of incidence advances over the Bragg peak region, the antinodal pattern moves inward and eventually the antinodes are within the Pt layers at the falling edge of the Bragg peak. The effect of this movement of the antinodal pattern within the multilayer produces an incident angle-dependent fluorescence yield from $\mathrm{Pt}$ and $\mathrm{Fe}$ atoms. Analysis of this angle-dependent fluorescence yield provides information about atomic distribution in the multilayer.

Variation of Pt and Fe fluorescence yields-experimental data and the fitted curves-are shown in Fig. 5. The incident angle dependence of fluorescence yield has been modeled following Refs. 5 and 21. The inset shows three curves marked 1,2, and 3. Curves 1 and 2 represent theoretical $\mathrm{x}$-ray field intensities integrated over the $\mathrm{C}$ and Pt layers, respectively. In the dipole approximation, the fluorescence yield from an atom is proportional to the field intensity on the atom. So the fluorescence yield from $\mathrm{C}$ and Pt layers should resemble curves 1 and 2, respectively. When all the $\mathrm{Fe}$ atoms are in the $\mathrm{C}$ layers, $\mathrm{Fe}$ fluorescence yield would also follow curve 1. On the other hand, if all $\mathrm{Fe}$ atoms are in the Pt layers, Fe fluorescence yield would follow curve 2. $50 \% \mathrm{Fe}$ in Pt layers and the other 50\% in C layers, with uniform depth profiles in the respective layers, would give rise to a fluorescence yield curve resembling curve 3. Analysis of the shape of the Fe fluorescence yield curve provides the relative concentrations of $\mathrm{Fe}$ in the $\mathrm{Pt}$ and $\mathrm{C}$ layers. Experimental $\mathrm{Pt} L_{\alpha}$ and $\mathrm{Fe} K_{\alpha}$ fluorescence yield versus the angle of incidence over the first order Bragg peak from all the samples are shown in Fig. 5. (The fluorescence yield curves have been given the required corrections as discussed in Refs. 5 and 21.) For the samples B-F, reflectivity (already in Fig. 2) has not been plotted and the fluorescence yield data have been vertically shifted for clarity. We fit the fluorescence yield data based on the model described earlier in Ref 21. For the XSW analysis, we have kept the thickness and roughness parameters, as extracted from the XRR analysis, fixed. However, slight variation of electron density was required to fit the fluorescence data. We first discuss Pt fluorescence yield. If the fluorescence yield can be fitted assuming the presence of Pt only in the Pt layers and within the broadened interface (broadening due to interface roughness, represented by an error function), we call this situation to have a coherent fraction $f_{c}$ equal to $1 .^{21}$ The presence of a small fraction of total $\mathrm{Pt}$ dissolved in uniform distribution in $\mathrm{C}$ layers corresponds to $f_{c}<1$. The coherent fractions of $\mathrm{Pt}$, obtained from the best fit to Pt fluorescence data, are shown in Table I. The coherent fraction of Pt decreases from 0.93 in the virgin sample $\mathrm{V}$ to 0.83 in the ion-irradiated sample $\mathrm{C}$, which indicates that $\mathrm{Pt}$ concentration in $\mathrm{C}$ layers increases with ion fluence. That is, ion-beam induced atomic mixing takes place up to sample C. At higher ion fluences, i.e., for samples $\mathrm{D}$ and $\mathrm{F}$, there is demixing of $\mathrm{Pt}$ atoms from $\mathrm{C}$

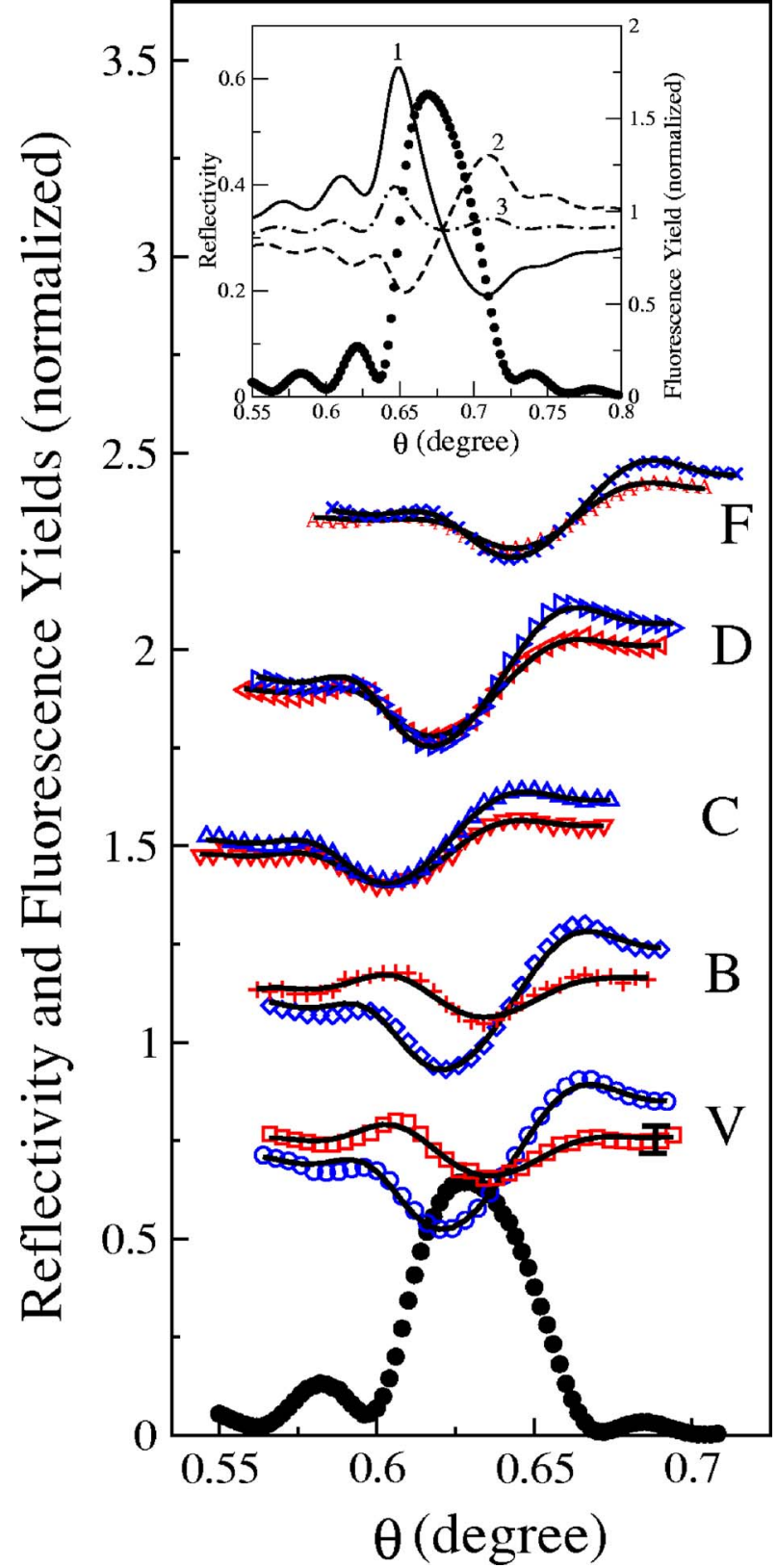

FIG. 5. Experimental Pt $L_{\alpha}$ (blue symbols, V-open circle, B-diamond, C-triangle up, D-triangle right, F-cross) and $\mathrm{Fe} K_{\alpha}$ (red symbols, V-square, B-plus, C-triangle down, D-triangle left, F-character A) fluorescence yield vs angle of incidence $\theta$ over the first order Bragg reflection and the fitted theoretical curves (black solid curve) for the virgin $(\mathrm{V})$ and the irradiated (B-F) periodic $\mathrm{Pt}(\mathrm{Fe}) / \mathrm{C}(\mathrm{Fe})$ multilayer samples. Fluorescence data have been normalized at $\theta=2.9^{\circ}$. Data for the irradiated samples are vertically shifted up for clarity. Inset figure: theoretical plots showing the first order Bragg peak and $\mathrm{x}$-ray field intensities on $\mathrm{Fe}$ in a $\mathrm{Pt}(\mathrm{Fe}) / \mathrm{C}(\mathrm{Fe})$ multilayer for three cases. Reflectivity (filled circle), theoretical x-ray field intensity on $\mathrm{Fe}$ when $\mathrm{Fe}$ is uniformly distributed over the $\mathrm{C}$ layers (1 solid line), field intensity when $\mathrm{Fe}$ is uniformly distributed over the Pt layers (2 dashed line), field intensity when $\mathrm{Fe}$ is in uniform distributions both in $\mathrm{Pt}$ and $\mathrm{C}$ layers with $50 \%$ of total $\mathrm{Fe}$ in each layer (3 dashed-dotted line)

layers (coherent fraction increases again to 0.87 in samples D and F). Thus Pt fluorescence yield also shows the same trend of mixing and demixing effect of $\mathrm{Pt}$ and $\mathrm{C}$ atoms depending on ion fluence, as inferred from the XRR analysis. We will discuss Fe fluorescence yield in Sec. III B.

Variation of several parameters as a function of ion fluence is shown in Fig. 6. We notice that up to a fluence of 


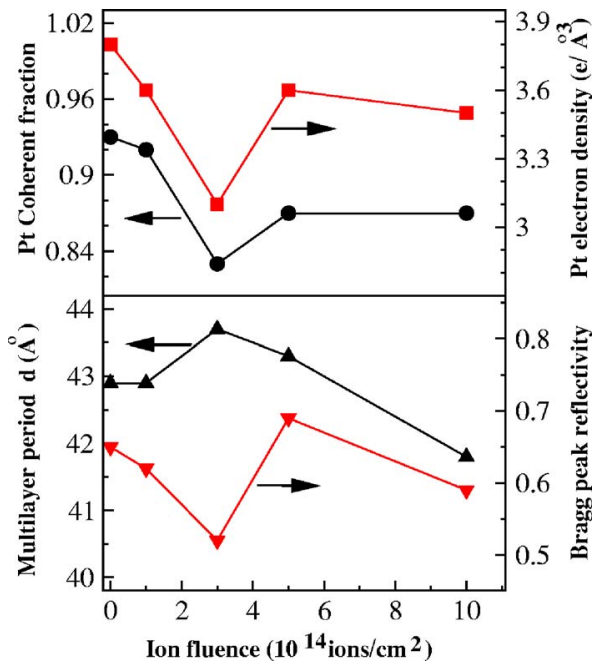

FIG. 6. Variation of several parameters as a function of ion fluence.

$3 \times 10^{14}$ ions $/ \mathrm{cm}^{2}$ there is a certain trend which suddenly reverses at that fluence. As explained earlier ballistic mixing across the $\mathrm{Pt} / \mathrm{C}$ interfaces occur up to this fluence, which we may call a threshold fluence, $\phi_{t}$. At $\phi>\phi_{t}$ chemically driven processes dominate and all the parameters shown in Fig. 6, along with several other parameters shown in Table I, reverse the trend. The trend observed here for $\phi<\phi_{t}$ is in agreement with our earlier observation and simulation results. ${ }^{5}$ Many parameters change trend for $\phi>\phi_{t}$. At high ion fluence the density of point defects created in the medium is high. These point defects are mobile and diffusional processes are enhanced by irradiation. Thermally activated diffusive processes then tend to restore the system to its equilibrium state. When $\phi \gg \phi_{t}$, for example $\phi=1 \times 10^{15} \mathrm{ions} / \mathrm{cm}^{2}$, anomalous behavior is observed from Fig. 6. At this fluence the dominating chemically driven processes destroy the integrity of the multilayer, as corroborated by the XTEM micrograph in Fig. 4(F); even new length scales evolve in the transformed microstructure. This aspect was discussed in details in our earlier work. ${ }^{24}$

\section{B. Atomic migration}

We now discuss the evolution of $\mathrm{Fe}$ fluorescence yield (FY) curve with ion irradiation. In Fig. 5 we notice that for the virgin sample $\mathrm{V}$, the $\mathrm{Fe} K_{\alpha} \mathrm{FY}$ at the angular position of the rising edge of the Bragg peak is higher. The Fe $K_{\alpha} \mathrm{FY}$ curve shows similarity to the theoretical curve 3 of the inset, which indicates a nearly uniform distribution of $\mathrm{Fe}$ in both $\mathrm{Pt}$ and $\mathrm{C}$ layers. For the virgin sample (V), fitting of the $\mathrm{Fe} K_{\alpha}$ fluorescence yield data to theory shows that $55 \%$ of $\mathrm{Fe}$ atoms are in the Pt layers and the remaining $45 \%$ are in the $\mathrm{C}$ layers. ${ }^{25}$ The last column in Table I shows the fraction of $\mathrm{Fe}$ atoms in the Pt layers as coherent fraction. For sample B, Fe $K_{\alpha} \mathrm{FY}$ intensities at both edges of the Bragg peak are approximately equal. For higher ion fluences (samples $\mathrm{C}-\mathrm{F}$ ), the intensity is always higher on the high-angle edge, indicating an increasing Fe concentration in the Pt layers. In fact, the shapes of the Fe $K_{\alpha}$ FY curves for the samples D and F nearly correspond to the shapes of the $\mathrm{Pt} L_{\alpha} \mathrm{FY}$ curves. This indicates that on passing from $\mathrm{V}$ to $\mathrm{F}, \mathrm{Fe}$ atoms in the $\mathrm{Pt}(\mathrm{Fe}) /$

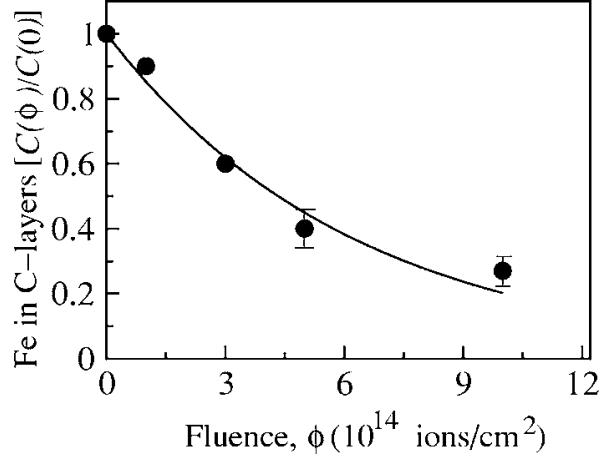

FIG. 7. Variation of the normalized Fe concentration in the $\mathrm{C}$ layers with ion fluence.

$\mathrm{C}(\mathrm{Fe})$ multilayer have been considerably redistributed with higher ion fluences, generating a higher concentration of $\mathrm{Fe}$ in the Pt layers (from $55 \%$ of total $\mathrm{Fe}$ in sample $\mathrm{V}$ to $88 \%$ of total $\mathrm{Fe}$ in sample $\mathrm{F}$ ). This corresponds to $45 \%$ of total $\mathrm{Fe}$ in the $\mathrm{C}$ layers in the sample $\mathrm{V}$ to $12 \%$ in the $\mathrm{C}$ layers in sample F. Fitted values of the Fe coherent fraction are shown in the last column of Table I. This Fe migration from $\mathrm{C}$ to Pt layers are due to the fact that the $\mathrm{Fe}-\mathrm{C}$ system is almost immiscible (heat of mixing $\left.\Delta H_{m}^{1: 1}=35.3 \mathrm{~kJ} / \mathrm{mol}\right)^{24}$ under equilibrium condition and the carbide phases are metastable. Consequently phase separation is predictable between $\mathrm{Fe}$ and $\mathrm{C}$ due to ion irradiation. On the other hand, $\mathrm{Fe}$ and $\mathrm{Pt}$ can form alloys at all compositions.

The change in the normalized Fe concentration in the $\mathrm{C}$ layers with ion fluence in irradiation is shown in Fig. 7. This dependence of $\mathrm{Fe}$ concentration can be described well by

$$
\mathrm{C}(\phi)=\mathrm{C}(0) \times \exp (-\sigma \phi),
$$

where $\phi$ is the ion fluence (ions $\left./ \mathrm{cm}^{2}\right), \mathrm{C}(0)$ is the $\mathrm{Fe}$ concentration in the virgin sample, $\mathrm{C}(\phi)$ is the Fe concentration after irradiation at a fluence $\phi$, and $\sigma$ may be called ion beam induced atomic migration cross section. $\sigma$ is a parameter determined by the material and the kind of the ion beam and ion energy, and in turn by the energy deposited per unit depth from the ion to the irradiated material. Equation (1) has been derived using an approach similar to that in Ref. 26. The normalized $\mathrm{Fe}$ concentration in $\mathrm{C}$ layers, $\mathrm{C}(\phi) / \mathrm{C}(0)$, as a function of ion fluence (Fig. 7) appears to obey Eq. (1). The parameters obtained by fitting the $\mathrm{C}(\phi) / \mathrm{C}(0)$ versus $\phi$ data in Fig. 7 to Eq. (1) are $\mathrm{C}(0)=1.00 \pm 0.05$ and $\sigma=(1.6 \pm 0.2)$ $\times 10^{-15} \mathrm{~cm}^{2}$.

Ion-beam mixing or demixing in multilayers can be understood from ballistic mixing (temperature independent mixing) and ion-irradiation-enhanced diffusion. In the case of elements with large positive heat of mixing like Pt and C, the two mechanisms operate in opposite directions. Ballistic effects are always present and act as a mixing mechanism in the initial stages of the collision. For radiation enhanced diffusion, the energy lost by the primary ion generates atomic relocations in a cascade of nuclear collisions. The energy of the recoil atom is thermalized by secondary collisions down to kinetic energies of a few electron-volts, comparable with chemical potentials in solids. At these energies the random walk atomic relocations can be chemically biased. 


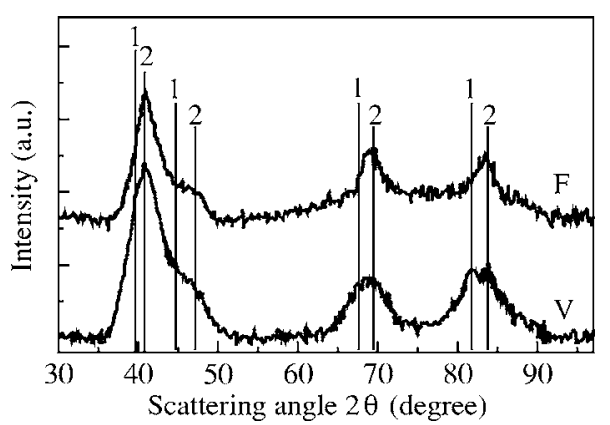

FIG. 8. GIXRD patterns from the virgin sample (V) and sample irradiated at the highest fluence $(\mathrm{F})$ measured with $\mathrm{Cu} K_{\alpha}$ x rays. The positions of the diffraction lines are indicated: Pt (1), FePt (2).

At low fluences, when there is no spatial superposition of the cascades, only short-range relocation within the collision cascade is possible so that the initial ballistic mixing is partially recovered by chemically driven relocation within the cascades. The role of chemical driving force is enhanced for high fluences when atomic relocations over extended regions due to the spatial superposition of cascades promote long range atomic migration and phase segregation where the components are immiscible. Thus we have mixing between $\mathrm{Pt}$ and $\mathrm{C}$ at low fluences and demixing effect at higher fluences due to the two competitive processes discussed earlier. Fe migration from $\mathrm{C}$ to $\mathrm{Pt}$ layers is understood in the light of the Fe-C and Fe-Pt phase diagram. We have explained the demixing effect of $\mathrm{Pt}$ and $\mathrm{C}$ and $\mathrm{Fe}$ migration due to ion irradiation in Ref. 24 by calculating heat of mixing and effective diffusion coefficients for the corresponding binary systems.

GIXRD results from the virgin sample and the sample irradiated at the highest fluence $(\mathrm{F})$ are shown in Fig. 8. In the GIXRD pattern from the virgin sample, the positions marked " 1 " are at the expected peak positions for diffraction from pure Pt and the positions marked "2" are from FePt (these peak positions are also consistent with a $2.5 \%$ contracted Pt. This contration may be a consequence of alloy formation with $\mathrm{Fe}$ ). As the virgin sample, $\mathrm{V}$, contains $\mathrm{Fe}$ in the Pt layers, we observe contributions from both $\mathrm{Pt}$ and FePt in the diffraction. The existence of FePt in the virgin sample is also consistent with a small hysteresis loop observed in Kerr rotation for this sample. ${ }^{22}$ From Fig. 8 it is clear that Pt diffraction peak intensities decrease and $\mathrm{FePt}$ diffraction peak intensities increase in the irradiated sample, F, compared to the virgin sample, V. This change in XRD pattern also indicates $\mathrm{Fe}$ migration into the $\mathrm{Pt}$ layers. This migration of $\mathrm{Fe}$ atoms into Pt layers gives rise to formation of magnetic $\mathrm{FePt}$ alloy particles giving rise to magnetism in the ion irradiated multilayers. ${ }^{22,24}$ The increase in $\mathrm{Fe}$ concentration in the Pt layers with increasing fluence also gives rise to increased magnetic strength in these ion-irradiated multilayers as reflected in the increasing coercive field in the hysteresis loop in Kerr rotation. ${ }^{22}$ About the FePt particles it is natural to ask whether they are in the tetragonal $\left(L 1_{0}\right)$ phase. As the diffraction peaks are broad, from the FePt peaks in the XRD pattern it cannot be ascertained whether FePt is in tetragonal $\left(L 1_{0}\right)$ phase or in the cubic phase.

\section{Surface-directed Fe migration}

In Sec. III B we have discussed $\mathrm{Fe}$ migration from $\mathrm{C}$ to Pt layers. We have not discussed whether Fe migrates symmetrically outward and inward to the nearest Pt layers, or there is a directional preference for Fe migration. Is it possible to determine this directional preference for $\mathrm{Fe}$ atoms migrating within a $2 \mathrm{~nm}$ thin $\mathrm{C}$ layer? The answer is yes. Our detailed analysis shows that this Fe migration is not symmetric about the $\mathrm{C}$ layers; $\mathrm{Fe}$ migration is asymmetric with preferential migration into the $\mathrm{Pt}$ layers toward the surface. Here we explain how this directional preference of atomic migration in a nanostructured periodic multilayer can be determined. We refer to the XSW experiments again to detect directional preference for atomic migration. To demonstrate this let us assume there is one atomic layer of $\mathrm{Fe}$ at the center of all $\mathrm{C}$ layers in the multilayer. This Fe layer is exposed to the maximum $\mathrm{x}$-ray intensity (antinode) when the angle of incidence corresponds to the rising edge of the Bragg peak, while at the falling edge of the Bragg peak the Fe layer is exposed to the minimum of x-ray intensity (node). As the intensity of emission of Fe $K_{\alpha}(6.4 \mathrm{keV})$ fluorescence is proportional to the intensity of incident $\mathrm{x}$ rays $(14 \mathrm{keV})$ the $\mathrm{Fe}$ atoms are exposed to, the angular variation of the $\mathrm{Fe} K_{\alpha}$ fluorescence yield will be given by the curve marked " 0.5 " in Fig. 9(a). If the Fe layer migrates inward, say to positions $0.4,0.3,0.2,0.1$, and 0 (middle of the nearest inner Pt layer), the $\mathrm{Fe} K_{\alpha}$ fluorescence yield curve would change accordingly, as marked in Fig. 9(a). The case of outward movement of $\mathrm{Fe}$ is illustrated in Fig. 9(b). We note that the way the shape of the curves changes from position 0.5 for inward [Fig. 9(a)] and outward [Fig. 9(b)] migration of Fe is quite different. Thus a measurement of the evolution of the Fe $K_{\alpha}$ fluorescence yield curve can distinguish between inward and outward migration of Fe. Comparing this change of theoretical fluorescence yield curves with the evolution of the experimental Fe $K_{\alpha}$ fluorescence yield curve from samples V to $\mathrm{F}$ in Fig. 5 one can confirm the outward movement of $\mathrm{Fe}$ atoms. The inward migration can be easily ruled out as the experimental Fe $K_{\alpha}$ fluorescence profiles (Fig. 5) never move through the shapes marked " 0.3 " or " 0.2 " in Fig. 9(a). These aspects have been explained in more detail elsewhere. ${ }^{27}$

The outward movement of $\mathrm{Fe}$ is also indicated in the magnetic force microscopy (MFM) measurements. As MFM is sensitive to the outer region of the sample, magnetic domain structure is observed in the irradiated samples, while the virgin sample does not show magnetic domains although magneto-optical Kerr effect measurements, which probes a larger depth, shows a small hysteresis loop for the virgin sample. $^{22}$

Why is the migration of $\mathrm{Fe}$ directed toward the surface (outward) of the multilayer? For binary alloys the prediction of surface segregation is based on the value of heat of segregation $(Q)$. If $Q$ is large and positive then segregation should occur. For the $\mathrm{Pt}(\mathrm{Fe})$ [solvent(solute)] system, Burton and co-workers failed to find any evidence of segregation of Fe to the surface of polycrystalline Pt by annealing the samples up to $1200{ }^{\circ} \mathrm{C}$ under ultrahigh vacuum condition. ${ }^{28}$ The predicted heat of segregation of Fe to the surface of Pt is 

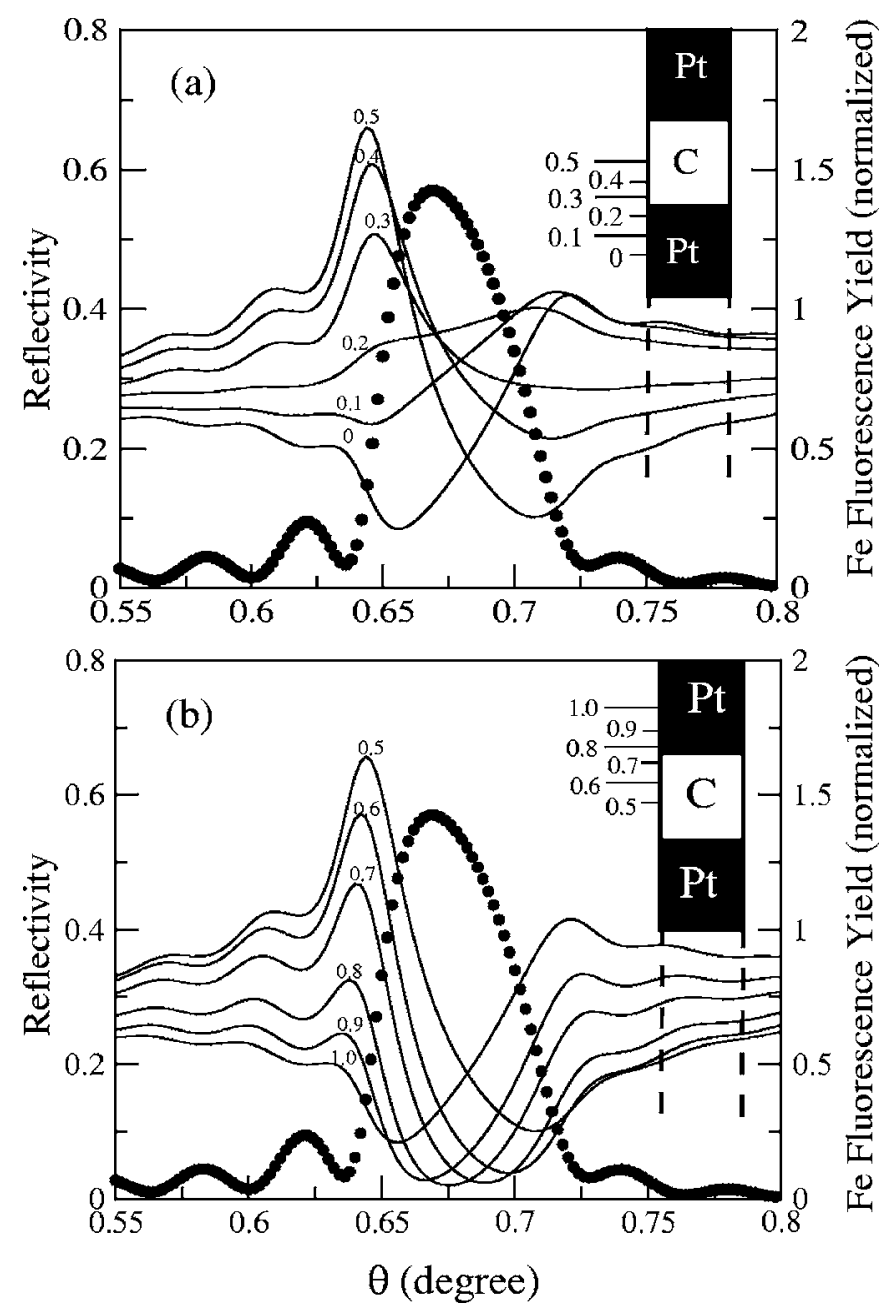

FIG. 9. Variation of $\mathrm{Fe} K_{\alpha}$ fluorescence yield curve over the first order Bragg peak from a $\mathrm{Pt} / \mathrm{C}$ multilayer containing an atomic layer of $\mathrm{Fe}$ at the center of a $\mathrm{C}$ layer. (a) when the layer of $\mathrm{Fe}$ migrates inward from position 0.5 (center of a $\mathrm{C}$ layer) to $0.4,0.3, \ldots, 0.0$ (center of a Pt layer) and (b) outward to $0.6,0.7, \ldots, 1.0$ (center of a Pt layer). For inward and outward movements of Fe, Fe fluorescence yield evolves in a distinctly different way.

below $2 \mathrm{kcal} / \mathrm{mol} .{ }^{29}$ This low value of $Q$ was their explanation for the absence of $\mathrm{Fe}$ surface segregation in Pt. But when they exposed their sample to a poorer vacuum-1 $\times 10^{-7}$ Torr of $\mathrm{O}_{2}$-at $700{ }^{\circ} \mathrm{C}$, they observed surface segregation of $\mathrm{Fe}$ because $\mathrm{O}_{2}$ can draw Fe to the surface of Pt. ${ }^{28} \mathrm{In}$ our case during ion irradiation of our samples, the pressure in the irradiation chamber was about $1 \times 10^{-7}$ Torr. We believe the presence of $\mathrm{O}_{2}$ in the chamber at this pressure was responsible for dragging Fe toward the surface of Pt. Once this segregation starts, Fe atoms migrate in the outward direction throughout the multilayer stack. This appears to be the reason for outward migration of $\mathrm{Fe}$ atoms in our ion-irradiated $\mathrm{Pt}(\mathrm{Fe}) / \mathrm{C}(\mathrm{Fe})$ multilayers.

\section{CONCLUSIONS}

We have carried out irradiation of $\mathrm{Pt}(\mathrm{Fe}) / \mathrm{C}(\mathrm{Fe})$ multilayers, containing a small amount of $\mathrm{Fe}$, with $2 \mathrm{MeV} \mathrm{Au}^{2+}$ ions at different ion fluences. The samples were characterized by a combined XRR and XSW analysis. XTEM measurements were made to study microstructural changes of the irradiated samples. We have observed ion beam induced contraction as well as expansion in the multilayer period. Up to a certain fluence we have observed mixing between $\mathrm{Pt}$ and $\mathrm{C}$ atoms and an expansion of the multilayer period. At fluences higher than a threshold fluence $\left(\phi_{t}\right)$, the demixing effect has been pronounced and the process of expansion of multilayer period, along with many other parameters, is reversed. These phenomena have been explained in terms of two competitive processes including ballistic and chemically guided atomic movements. Ballistic ion mixing at the $\mathrm{Pt} / \mathrm{C}$ interface at low fluences is significantly counterbalanced by a chemically driven relaxation mechanism at high fluences. For the Pt/C system this results in demixing of the two materials at higher fluences. Upon ion irradiation of the multilayers, Fe atoms in the $\mathrm{Pt}(\mathrm{Fe}) / \mathrm{C}(\mathrm{Fe})$ multilayers preferentially migrate toward outward direction from $\mathrm{C}$ to Pt layers. Fe migration from the $\mathrm{C}$ to $\mathrm{Pt}$ layers is understood in the light of $\mathrm{Fe}-\mathrm{C}$ and $\mathrm{Fe}-\mathrm{Pt}$ phase diagrams. Outward migration of $\mathrm{Fe}$ is explained in terms of surface segregation. The Fe concentration in the $\mathrm{C}$ layers with increasing ion fluence follows an exponential decay. An ion-beam induced atomic migration cross section has been determined from this exponential decay. Migration of $\mathrm{Fe}$ from $\mathrm{C}$ to $\mathrm{Pt}$ layers gives rise to formation of FePt magnetic particles. The present study points to the possibility of using a focused ion beam of nanometer dimensions to create magnetic nanometer-size dots in a nonmagnetic medium. This is a goal for future high-density storage devices.

\section{ACKNOWLEDGMENTS}

The authors thank D. K. Goswami for help during irradiation, B. Satpati for the TEM, and Dr. F. Eichhorn for the GIXRD measurements. They also thank Prof. K. Yamashita for providing the as-deposited multilayer sample.

${ }^{1}$ D. B. McWhan, in Synthetic Modulated Structure, edited by L. L. Chang and B. C. Giesser (Academic, New York, 1985), Chap. 2, p. 43.

${ }^{2}$ M. B. Stearns, J. Appl. Phys. 55, 1729 (1984); also see the Proceedings of the International Conference on Magnetism, 1985, San Francisco (NorthHolland, Amsterdam, 1985).

${ }^{3}$ C. M. Falco and I. K. Schuller, Synthetic Modulated Structure Materials (Academic, New York, 1985), and references therein.

${ }^{4}$ S. S. P. Parkin, C. Chappert, and F. Herman, Europhys. Lett. 24, 71 (1993).

${ }^{5}$ S. K. Ghose, D. K. Goswami, B. Rout, B. N. Dev, G. Kuri, and G. Materlik, Appl. Phys. Lett. 79, 467 (2001).

${ }^{6}$ H. Zhang, R. W. Cochrane, Y. Huai, M. Mao, X. Bain, and W. B. Muir, J. Appl. Phys. 75, 6534 (1994).

${ }^{7}$ H. Laidler and B. J. Hickey, J. Appl. Phys. 79, 6250 (1996)

${ }^{8}$ M. Cai, T. Veres, S. Roorda, R. W. Cochrane, R. Abdouche, and M. Sutton, J. Appl. Phys. 81, 5200 (1997).

${ }^{9}$ M. Cai, T. Veres, S. Roorda, F. Schiettekatte, and R. W. Cochrane, J. Appl. Phys. 95, 1996 (2004).

${ }^{10}$ M. Cai, T. Veres, F. Schiettekatte, S. Roorda, and R. W. Cochrane, J. Appl. Phys. 95, 2006 (2004).

${ }^{11}$ C. Chappert et al., Science 280, 1919 (1998).

${ }^{12}$ B. D. Terris, L. Folks, D. Weller, J. E. E. Baglin, A. J. Kellock, H. Rothuizen, and P. Vettiger, Appl. Phys. Lett. 75, 403 (1999).

${ }^{13}$ D. Weller et al., J. Appl. Phys. 87, 5768 (2000).

${ }^{14}$ B. D. Terris, D. Weller, L. Folks, J. E. E. Baglin, A. J. Kellock, H. Rothuizen, and P. Vettiger, J. Appl. Phys. 87, 7004 (2000).

${ }^{15}$ R. Hyndman, P. Warin, J. Gierak, J. Ferre, J. N. Chaman, J. P. Jamet, V. Mathet, and C. Chappert, J. Appl. Phys. 90, 3843 (2001).

${ }^{16}$ P. Warin, R. Hyndman, J. Glerak, J. N. Chapman, J. Ferre, J. P. Jamet, V. Mathet, and C. Chappert, J. Appl. Phys. 90, 3850 (2001).

${ }^{17}$ G. J. Kusinski, K. M. Krishnan, G. Denbeaux, G. Thomas, B. D. Terris, and D. Weller, Appl. Phys. Lett. 79, 2211 (2001).

${ }^{18}$ G. J. Kusinski, G. Thomas, G. Denbeaux, K. M. Krishnan, and B. D. 
Terris, J. Appl. Phys. 91, 7541 (2002).

${ }^{19}$ M. J. Bonder, N. D. Telling, P. J. Grundy, C. A. Faunce, T. Shen, and V. M. Vishnyakov, J. Appl. Phys. 93, 7226 (2003).

${ }^{20}$ N. D. Telling, S. Langridge, R. M. Dalgliesh, P. J. Grundy, and V. M Vishnyakov, J. Appl. Phys. 93, 7420 (2003).

${ }^{21}$ S. K. Ghose and B. N. Dev, Phys. Rev. B 63, 245409 (2001).

${ }^{22}$ B. N. Dev et al., Microelectron. Eng. 83, 1721 (2006).

${ }^{23}$ Atomic concentration of $\mathrm{Fe}$ in the virgin sample has been determined from the relative intensities of $\mathrm{Fe} K_{\alpha}$ and $\mathrm{Pt} L_{\alpha}$ fluorescence peaks. This estimation has taken into account photoionization cross section for Fe $K$ shell and $\mathrm{Pt} L_{\mathrm{III}}$ subshell, their corresponding fluorescence yield, attenuation coefficients of incident photons and outgoing $\mathrm{Fe} K_{\alpha}$ and $\mathrm{Pt} L_{\alpha}$ photons in the multilayer, and an integration over the whole depth of the multilayer This provides a ratio of atomic concentrations of 39:61 for Fe:Pt. However, the Fe atoms are almost uniformly distributed over the whole depth of the multilayer and a fraction of the $\mathrm{Fe}$ atoms is present in $\mathrm{C}$ layers. For a Pt/C multilayer with $d=4.2 \mathrm{~nm}$ and $\Gamma=0.38$ (i.e. $1.6 \mathrm{~nm}$ Pt layers, 2.6 $\mathrm{nm} C$ layers: intended growth parameters) the ratio of the total number of $\mathrm{Fe}$ atoms $/ \mathrm{cm}^{2}$ to the sum of $\mathrm{Fe}, \mathrm{Pt}$, and $\mathrm{C}$ atoms $/ \mathrm{cm}^{2}$ turns out to be about 0.15 . Thus the average concentration of $\mathrm{Fe}$ is about 15 at. \%.

${ }^{24}$ S. Bera, B. Satpati, D. K. Goswami, K. Bhattacharjee, P. V. Satyam, and B. N. Dev, J. Appl. Phys. 99, 074301 (2006).

${ }^{25}$ The measured multilayer period is $d=4.2 \mathrm{~nm}$ where the thickness of $\mathrm{Pt}$ and $\mathrm{C}$ layers are 2.0 and $2.2 \mathrm{~nm}$, respectively. Using atomic densities of $\mathrm{Pt}$ $\left(6.6 \times 10^{22}\right.$ atoms $\left./ \mathrm{cm}^{3}\right)$ and amorphous $\mathrm{C}\left(1.0 \times 10^{23}\right.$ atoms $\left./ \mathrm{cm}^{3}\right), 45 \%$ of total $\mathrm{Fe}$ in $\mathrm{C}$ layers and 55\% in Pt layers lead to average layer compositions of $\mathrm{Fe}_{15} \mathrm{C}_{85}$ and $\mathrm{Fe}_{26} \mathrm{Pt}_{74}$, respectively. In the highest fluence irradiated sample $\mathrm{F}, 12 \%$ of total $\mathrm{Fe}$ in $\mathrm{C}$ layers and $88 \%$ in Pt layers lead to average layer compositions of $\mathrm{Fe}_{4} \mathrm{C}_{96}$ and $\mathrm{Fe}_{36} \mathrm{Pt}_{64}$, respectively.

${ }^{26}$ V. Hnatowicz, Nucl. Instrum. Methods Phys. Res. B 215, 162 (2004).

${ }^{27}$ S. Bera, K. Bhattacharjee, G. Kuri, and B. N. Dev, Phys. Rev. Lett. 98, 196103 (2007)

${ }^{28}$ J. J. Burton and R. S. Polizzotti, Surf. Sci. 66, 1 (1977).

${ }^{29}$ J. J. Burton and E. S. Machlin, Phys. Rev. Lett. 37, 1433 (1976). 